\title{
LA RESISTENCIA DESDE EL ARTE COMO PRÁCTICA CULTURAL EN LA CIUDAD
}

\section{RESISTANCE FROM ART \\ AS A CULTURAL PRACTICE IN THE CITY}

\author{
Olivia Fragoso Susunaga \\ Universidad Autónoma Metropolitana Unidad Azcapotzalco, México \\ oliviafragoso@azc.uam.mx
}

Recepción: 28 de febrero de 2019 • Aceptación: 22 de noviembre de 2019

RESUMEN

El texto aborda el fenómeno de la resistencia como práctica cultural en la ciudad. Para hacerlo se parte del concepto de la "objetividad entre paréntesis" que formula Maturana para comprender el fenómeno de la resistencia como práctica cultural. Se propone que este fenómeno pertenece al campo del discurso y que puede ser comprendido siguiendo las "prácticas semiótico discursivas" de Haidar. La explicación de la resistencia como fenómeno del arte se concibe entonces como práctica cultural, semiótico discursiva, la cual se entiende desde la óptica de la "estética política" de Rancière. Finalmente se plantea que, desde el enfoque de De Certeau, esta práctica es una táctica que, en el ámbito de lo cotidiano, siguen los actores ante las estrategias de dominio.

Palabras clave: estética, política, discurso.
ABSTRACT

The text is an approach to the phenomenon of resistance as a cultural practice in the city. To do so, we start from the concept of objectivity in parentheses formulated by Maturana to understand the phenomenon of resistance as a cultural practice. It is proposed that this phenomenon belongs to the field of discourse and that it can be understood following Haidar's semiotic discursive practices. The explanation of resistance as a phenomenon of art is then conceived as a cultural, semiotic discursive practice, which is understood from the perspective of Ranciere's political aesthetic. Finally, it is stated that, from De Certeau's approach, this practice is a tactic that, in the everyday sphere, the actors follow in the face of dominance strategies.

Keywords: aesthetics, politics, discourse. 


\section{INTRODUCCIÓN}

Trabajar sobre la ciudad en resistencia es ya un fenómeno de resistencia, más si se hace desde una mirada alterna. Es sencillo pensar en las experiencias vividas en la ciudad si se hace desde quienes cuentan la historia, de quienes tienen el control de la palabra, de quienes pueden rebelarse cuando algo no les gusta. También resulta sencillo pensar en las experiencias en la ciudad desde las maravillas que la modernidad, el progreso y el desarrollo han traído junto con la tecnología y los avances científicos. Pero, ¿qué sucede si se reflexiona sobre la versión no oficial?, ¿qué pasa con las experiencias con las que comprendemos el fenómeno cuando no perciben lo que se supone debe reconocerse en calles, edificios, monumentos, recorridos, tránsitos, gente, instituciones, prácticas, sitios y cada recoveco de la ciudad?

El presente trabajo es una reflexión sobre el fenómeno de la resistencia desde el arte como práctica cultural en la ciudad. En primer lugar, se define la relación resistencia-arte como una forma de enfrentarse al punto de vista hegemónico de la realidad. Para hacerlo se parte del concepto de la "objetividad entre paréntesis" que formula Maturana para comprender el fenómeno de la resistencia como práctica cultural. A continuación, se propone que este fenómeno pertenece al campo del discurso. Siguiendo las "prácticas semiótico discursivas", propuestas por la Dra. Haidar, se explican las operaciones y funcionamientos del arte como resistencia la cual como práctica semiótico discursiva se entiende desde la óptica de la "estética política" de Rancière. Finalmente se plantea que, desde el enfoque de De Certeau, esta práctica es una táctica que, en el ámbito de lo cotidiano, siguen los actores ante las estrategias de dominio.
La resistencia desde el arte como práctica cultural: un fenómeno transdisciplinario, complejo y de objetividad puesta entre paréntesis

Partimos del supuesto de que en la ciudad la resistencia desde el arte es una práctica cultural. En el orden en el que se formulan las ideas debería explicarse qué se entiende por ciudad, qué por resistencia y qué por práctica cultural. Sin embargo, antes de entrar en esta tarea es pertinente describir la óptica de la que se parte para comprender el fenómeno. Es pertinente mencionar que en este trabajo no pretendemos problematizar la definición de ciudad.

De acuerdo a Mumford (Homobono, 2003) la ciudad es "[...] un entorno ecológico, espacio de vida y arquitectónico; producto de una historia que proporciona las claves de su morfología y de su destino, vinculado al de la cultura." Creemos junto con el autor que la ciudad es un fenómeno complejo, por lo tanto, consideramos que puede ser comprendido mejor si se le aborda desde la perspectiva de la transdisciplinariedad.

Para hablar de resistencia seguimos la propuesta de Foucault. Guiraldo (2006), menciona, que para el autor el poder no es una propiedad de la que se pueda dar cuenta, nadie detenta el poder. Es una función que se presenta en el territorio de lo social. En ese mismo espacio y en el mismo sentido y como enfrentamiento a este se da la resistencia. En Foucault el poder no es absoluto ni fijo, se puede modificar en determinadas circunstancias de acuerdo con ciertas estrategias. La resistencia y el poder son una relación de fuerzas, lucha, oposición, como enfrentamientos. Para Foucault la resistencia, al enfrentarse al poder no es sólo su negación, sino un proceso social de transformación de la realidad.

La resistencia en la ciudad cumple una función transformadora al oponerse a las estrategias del poder. El arte puede ser un instrumento del poder y servir para replicar sus formas de 
dominio y de control. Sin embargo, en muchos casos el arte funciona como una estrategia de resistencia, enfrentando las formas que el poder asume mediante estrategias estéticas transformadoras que los habitantes de la ciudad recuperan de la vida cotidiana.

Al empezar a configurar la resistencia, desde el arte como práctica cultural en la ciudad, formulamos el supuesto de que para comprenderlo es mejor hacerlo desde la transdisciplinariedad y la complejidad para lo cual seguimos las ideas de Morin (1994 y 2009) y de Nicolescu (s.a.).

El siguiente problema que se presenta es plantear si este fenómeno se explica con la objetividad como forma de acercamiento a la realidad. Apoyándonos en Maturana, particularmente si entendemos a las prácticas culturales como experiencias, la objetividad resulta por lo menos ilusoria y difícil de comprender.

El fenómeno sobre el que reflexionamos, la resistencia desde el arte como práctica cultural, se aborda con diversos conceptos teóricos que provienen de distintas disciplinas y que deben entrar en un nivel dialógico para lograr los acuerdos necesarios para la comprensión del citado fenómeno por lo que se considera la propuesta de la Transdisciplinariedad.

[...] la transdisciplinariedad se interesa por la dinámica engendrada por la acción de varios niveles de realidad a la vez. El descubrimiento de esta dinámica pasa necesariamente por el conocimiento disciplinario. La transdisciplinariedad, no siendo nada más una nueva disciplina o una nueva hiperdisciplina, se nutre de la investigación disciplinaria, la cual, a su vez, se esclarece de una manera nueva y fecunda por el conocimiento transdisciplinario (Nicolescu s.a.: 36).

La sociología, la antropología cultural, la comunicación, los estudios visuales, la historia, la estética, la teoría del arte, el urbanismo, el diseño, la política, los estudios culturales, son campos del conocimiento que tendrían necesariamente que entrar en un nivel dialógico para poder comprender el fenómeno de las prácticas culturales.

Una de las principales características de este tipo de problemas, de acuerdo con Nicolescu es, a diferencia de la lógica clásica, la posibilidad de admitir el axioma del tercero incluido que permite la existencia de múltiples niveles de realidad. Esto significa que existe, por mencionar algunas, una realidad económica, una política, una social y una cultural, variables que operan tanto a nivel social como psicológico, filosófico, cultural y político.

La recursividad es una de las características de la visión de la transdisciplinariedad, que se encuentra presente en la resistencia desde el arte como práctica cultural. El fenómeno vuelve sobre sí mismo, el entorno influye sobre el sujeto y a su vez este influye sobre el primero, por lo que hay interacción constante y mutua afectación.

El tiempo como proceso irreversible. El tiempo no es una estructura con la que pueda comprenderse lo que sucede en la ciudad.

En el fenómeno de la resistencia, desde el arte como práctica cultural en la ciudad, opera la contradicción; la experiencia se desarrolla mediante la organización, al mismo tiempo que se produce mediante la desorganización, la desestructuración de los saberes y las nociones previas.

El tiempo es un componente fundamental en la experiencia de la resistencia desde el arte como práctica cultural en la ciudad. En la realidad, los mundos biológico, fisiológico y químico están en constante evolución. La vida fluye como proceso irreversible, lo mismo que la historia, ambas evolucionan, sin embargo están en una paradoja pues la vida conlleva la muerte, el origen implica de manera directa el fin y esta paradoja aplica a individuos, ideas, tradiciones, historias. Las experiencias vividas en la cultura misma se encuentran siempre en una paradoja, en una suerte de contradicción que en ocasiones se hace más 
evidente y a veces se oculta hasta casi pasar desapercibida, pero la contradicción está siempre presente.

Un problema complejo se diferencia de uno simple porque en la visión simplificadora:

[...] el pensamiento simplificante se fundó sobre la disyunción absoluta entre el objeto y el sujeto que lo percibe y concibe. Nosotros debemos plantear, por el contrario, el principio de relación entre el observador-conceptuador y el objeto observado, concebido. Hemos mostrado que el conocimiento físico es inseparable de la introducción de un dispositivo de observación, de experimentación (aparato, desglose, reja) y por esto incluye la presencia del observador-conceptuador en toda observación o experimentación (Morin, 2004: s.p.).

Al hablar de experiencias — como la resistencia desde el arte como práctica cultural en la ciudad entendida como fenómeno transdisciplinario y complejo-, siguiendo a Morin (2004), es fundamental contar con la presencia del observador-conceptuador y de la observación de segundo orden, dadas por el mismo sujeto que narra y/o analiza la experiencia. El conocimiento, desde el punto de vista de la experiencia estética, deja de considerarse como una relación sujeto-realidad-objeto para pensarse de acuerdo con Maturana (2002), como el resultado del proceso que se propone como explicación.

[...] el observador en la experiencia del observar, como el ser que distinguimos al observarnos en el observar. El observador no es un supuesto ontológico a priori. El observador aparece en la distinción del observar al hacer la pregunta por el observador y el observar. El observador es lo que queremos explicar y el observar es el instrumento con que queremos explicarlo (Maturana, 2002: 20).

Para el autor la cuestión central está en la configuración de la realidad, por lo que la forma en la que ésta se observa se convierte en un punto relevante de las discusiones académicas de la época contemporánea.

Para definir qué es una práctica seguimos la propuesta de Foucault, para quien la práctica está vinculada con el discurso; sin embargo de acuerdo con Castro, para Foucault no son exclusivamente una forma de reproducir discurso, sino que "[...] toman cuerpo en el conjunto de las técnicas, de las instituciones, de los esquemas de comportamiento, de los tipos de transmisión y de difusión, en las formas pedagógicas que, a la vez, las imponen y las mantienen" (Castro, 2004).

Si hablamos de arte como práctica cultural el problema entonces estriba en la identificación en el arte de ese conjunto de técnicas, del reconocimiento de las instituciones que se encuentran detrás de dicho fenómeno. En este trabajo no pretendemos hablar de la manera en la que en un caso específico operan las prácticas, sino exponer que el arte puede comprenderse como una práctica vinculada con un discurso, que tiene una determinada forma de transmisión, difusión y formas pedagógicas.

Si en esta práctica lo que nos ocupa es la experiencia, habría que tener claro también al observador como una entidad biológica, inmerso en un proceso de conocimiento, para el que emplea un lenguaje que se encuentra articulado por las demás prácticas de la cultura y del discurso, y a su vez se encuentra en un campo complejo, transhistórico, ético y social. En este proceso el observador, dice Maturana (2002), para explicar la realidad sigue dos caminos que se dan de manera simultánea en las distintas esferas que lo atraviesan.

El primer camino es:

[...] ciego y sordo [...] las entidades asumidas como existentes con independencia de lo que el observador hace, así como esas entidades que surgen como constructos de éstas, constituyen lo real y cualquier otra cosa es una ilusión. En otras palabras, en este camino 
explicativo sostener que una afirmación dada es una ilusión, es rehusar su realidad, y negar su validez (Maturana, 2002: 21).

Como podemos suponer, este camino plantea la existencia de una sola forma de comprender la realidad, y de observarla, cualquier desviación a la regla es una anomalía.

Existe por otra parte un camino al que denomina de "objetividad entre paréntesis" en el que de acuerdo con el autor en la observación de la realidad hay una aceptación del ser humano como un sistema vivo, con capacidades cognitivas que son alteradas por la evolución del ser humano hacia la muerte y que como observadores reconocen en tanto:

[...] sistemas vivientes, particularmente sus incapacidades para distinguir en la experiencia lo que en la vida diaria distinguimos como percepción e ilusión [...] El uso que nosotros hacemos en la vida diaria de las palabras mentira y error, revela esto, y la palabra hipocresía demuestra que nosotros usamos nuestra incapacidad para distinguir en la experiencia entre percepción e ilusión para la manipulación de nuestras relaciones interpersonales (Maturana, 2002: 23).

El autor menciona que los seres humanos, al igual que los animales, carecen de la capacidad para distinguir percepción e ilusión, y ese hecho imposibilita al observador para referirse a los objetos como si "existieran independientemente" de lo que el observador hace; este en tanto especie animal y como cualquier otro ente biológico, carece de fundamentos tanto fisiológicos como cognitivos como para hacer tales afirmaciones. "Su acuerdo no da validez operacional a una distinción que ninguno de ellos pueda hacer individualmente" (Maturana, 2002: 23).

Al seguir la ruta de la "objetividad entre paréntesis", el observador existe a partir de las "[...] operaciones de distinciones en su praxis de vivir en el lenguaje" (Maturana, 2002: 23). Para seguir ese camino, el observador, menciona el autor, debe tener en principio conciencia de su condición biológica; al mismo tiempo que reconocimiento del funcionamiento de las operaciones discursivas de la práctica del lenguaje. Por otra parte, es fundamental el dominio de la realidad en tanto que dominio de coherencias operacionales, es decir, cómo operan las condiciones del lenguaje de la realidad que se observa y la forma en la que ésta se pone en praxis, es decir, las reglas del juego de la vida en la que la realidad observada se encuentra como sistema integrada.

La recursividad, en tanto que fenómeno complejo, es recuperada por Maturana para dar cuenta de las reformulaciones de la praxis de la realidad observada y de esta manera procurar la coherencia en la integración de la observación. Finalmente los distintos niveles de realidad que como sistema complejo existen, pueden ser usados para comprender, explicar, generar o distinguir los distintos dominios de realidad que explican diferentes aspectos de la praxis del vivir.

Considerar el fenómeno de la resistencia desde el arte como práctica cultural en la ciudad desde la objetividad entre paréntesis de Maturana nos lleva a plantear que el observador del fenómeno se encuentra en una condición biológica, por lo que las operaciones de distinción deberían considerar el campo de la estética realizadas en donde se dan operaciones discursivas de la práctica del lenguaje, que habrá que reconocer para elaborar las distinciones pertinentes de dicha praxis en la comprensión de la realidad en la que la estética y la ciudad se encuentran.

\section{El fenómeno de la resistencia desde el arte como práctica cultu- ral, problema relativo al campo del discurso}

Como vimos en el apartado anterior, el fenómeno de la resistencia como práctica cultural se ubica en la ruta de la objetividad entre paréntesis formulada por Maturana como praxis que "[...] el observador trae a la mano los objetos que él o ella distingue con sus operaciones de distinción, como distinciones de distinciones en el lenguaje" (Maturana, 2002: 24). Con 
fundamento en esta idea recuperamos el concepto de "prácticas discursivas" desarrollado en La arqueología del saber de Foucault (1972), el concepto de "prácticas sociales" de Bourdieu (1990) y el concepto de "prácticas semiótico discursivas" de Haidar (2006). Haidar recupera los dos anteriores y explica, desde un enfoque de la transdisciplina y la complejidad, el funcionamiento de la práctica cultural basado en las materialidades discursivas, elementos fundamentales para comprender el fenómeno.

Las [...] prácticas contienen: materialidades, funcionamientos, operaciones peculiares que inciden de manera determinante para la producción y reproducción de la vida socio-histórico-políticocultural de los sujetos [...] [En un sentido extenso es la] Comprensión del funcionamiento de lo cultural, de lo social, de lo histórico, de lo ideológico y de lo político en las distintas prácticas semiótico-discursivas, y que permiten dar cuenta de la complejidad analítica de los objetos de estudio en nuestro mundo contemporáneo (Haidar, 2006: 46).

La autora en su modelo enumera una serie de materialidades de las que elegimos la estética, la retórica, la cultural, la social, la ideológica, la del poder, la política, la lógico-filosófica, la del simulacro y la histórica.

Estas materialidades, desde la perspectiva teórica señalada, se convierten en categorías de análisis que deben ser desarrolladas ampliamente y correlacionadas con la realidad para dar cuenta de la experiencia estética en la operación del discurso. No es el propósito de este trabajo hacer una aplicación del concepto sino formular su pertinencia en la comprensión del fenómeno. Se señala una selección de las materialidades porque la misma autora menciona la pertinencia de elegir aquella o aquellas que se consideren adecuadas para la el análisis y explicación de los distintos fenómenos del discurso, e incluso, la autora, formula la posibilidad de ampliar las categorías para dar cuenta de la forma en la que las prácticas culturales discursivas funcionan y operan.

De acuerdo con lo expuesto la comprensión del arte como práctica cultural implicaría identificar el discurso subyacente y para ello la identificación de la esfera estética, la retórica, la cultural, la social, la ideológica, la del poder, la política, la lógicofilosófica, la del simulacro y la histórica nos permiten tener los elementos suficientes para determinar el funcionamiento del discurso del poder o el arte como práctica cultural en resistencia.

Como ejemplo, una muestra de activismo artístico en la ciudad de Ai Weiwei con su obra Study of Perspective una serie de fotografías que para comprenderlas hay que dar cuenta de las materialidades en las que funciona la práctica cultural.
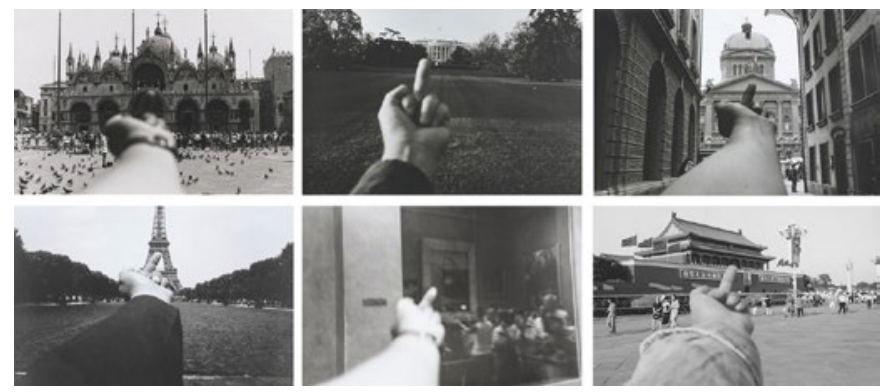

Imagen 1. Study of Perspective. Ai Wei Wei.1995-2003.

Habría que tener en cuenta para la comprensión del arte, como práctica cultural, las condiciones de producción, circulación y recepción, en las cuales se pueden observar las materialidades. En el caso de la obra de Ai Weiwei al analizar el poder y la ideología como materialidades discursivas habría que considerar:

a. Las condiciones de posibilidad de emergencia de los discursos y de las distintas semiosis. 
b. La relación entre formación socio-históricocultural-política, formación ideológica/ hegemónica y formación discursivo-semiótica.

c. Las formaciones imaginarias en las prácticas semiótico-discursivas.

d. La relación discurso/semiosis y coyuntura.

e. Las gramáticas de producción y recepción de las semiosis y de los discursos.

f. La aceptabilidad de los discursos y de las semiosis.

g. Los procesos de interdiscursividad, intertextualidad y de intersemiosis.

h. La situación y las interacciones comunicativas de las prácticas semiótico-discursivas (Haidar, 2006: 103).

Es evidente que habría que tener un conocimiento lo más completo posible de la realidad en la que el fenómeno se inscribe; como lo apuntó Maturana, es una necesidad en el trayecto de la objetividad entre paréntesis el saber en qué situación la entidad biológica va a experimentar las experiencias. Las condiciones de producción, circulación y recepción.

Es también importante tener en cuenta la forma en la que las operaciones discursivas funcionan. En el caso de Study of Perspective habría que resolver en relación al poder y la ideología:

En la selección de los objetos semiótico-discursivos, el poder los prohíbe o los impone, es decir, obliga su presencia o su ausencia -la exclusión. Del mismo modo, el poder y la ideología funcionan en las esquematizaciones de los objetos semiótico discursivos, en sus construcciones... En consecuencia, estos procesos pueden aparecer en los discursos bajo varias formas, o se ocultan en los implícitos, de modo indirecto, con eufemismos. Las formaciones imaginarias están regidas por las formaciones ideológicas que, a su vez, implican formaciones discursivas. Las formaciones imaginarias, que están automáticamente en toda producción semiótico-discursiva, según la propuesta de Pêcheux derivan, por lo tanto, de las posiciones que ocupan los sujetos en la estructura social (Haidar, 2006: 92).

Para la autora las materialidades del poder y de la ideología son fundamentales en la articulación de los discursos, evidentemente siguiendo la línea de Foucault. No obstante, como ya lo mencionamos, es posible formular otras categorías que permitan la explicación específica del fenómeno; en el caso de la obra de referencia como suponemos que está en resistencia habría que pensar en su pertenencia a la práctica del militarismo artístico o al arte militante, lo que implica una materialidad política importante. Para nuestro punto de vista, la estética y la política son categorías fundamentales para comprender el arte como práctica cultural en la ciudad ya sea que pertenezca a la resistencia o sirva como un instrumento del poder.

\section{Estética y política}

Existe un discurso político implícito en la idea de prácticas culturales, vamos a partir de ese postulado y del hecho de que dicho discurso está vinculado con un valor. Para empezar, tendríamos que aclarar a qué nos referimos cuando hablamos de política, y posteriormente relacionar tal definición con el concepto de valor.

En la definición de política consideramos partir de un autor que ha relacionado la política con la estética y ha dado cuenta de numerosas experiencias en este campo.

La expresión "política de la literatura" implica que la literatura hace política en tanto literatura. Supone que no hay que preguntarse si los escritores deben hacer política o dedicarse en cambio a la pureza de su arte, sino dicha pureza misma tiene que ver con la política. Supone que hay un lazo esencial entre la política como 
forma específica de la práctica colectiva y la literatura como práctica definida del arte de escribir (Rancière, 2011: 15).

Si la literatura hace política en tanto que literatura, mutatis mutandi entenderemos que las prácticas culturales hacen política en tanto que prácticas culturales. Dado que la política y la cultura son formas específicas de prácticas colectivas y construcciones ideológicas, los grupos humanos les asignan ciertos valores. El autor explica, valiéndose de la literatura, conceptos que permiten la comprensión de la experiencia en las relaciones entre política y estética.

[M]e interesa la literatura no como una disciplina sino, por el contrario, como principio de desclasificación de los discursos. Por lo tanto, no creo que haya método literario o competencia literaria específica. Para mí, la literatura no es un arte o un dominio cerrado sobre sí mismo, a la espera de especialistas que vengan a despejar sus leyes y a permitir la apreciación de sus obras. [La literatura] es un régimen histórico del arte de escribir que precisamente se caracteriza por la abolición de las reglas de las artes poéticas, por el hecho de que no hay cerramiento del sistema, y porque no hay ni siquiera oposición entre una razón de ser de las ficciones y una razón de ser de los hechos. La literatura designa para mí una apertura de las fronteras entre los discursos y no necesita de expertos para esa apertura [... por lo tanto, yo no me he ocupado de producir una teoría de la literatura que brinde instrumentos que permitan despejar las reglas y explicar en general y transmitir las obras literarias [...] (Rancière citado en Bejarano, 2010: 170).

Siguiendo a Rancière cuando se habla de política:

Se la confunde a menudo con el ejercicio del poder y la lucha por el poder. Pero no basta con que haya poder para que haya política [...] La política es la constitución de la esfera de experiencia específica donde se postula que ciertos objetos son comunes y se considera que ciertos sujetos son capaces de designar tales objetos y de argumentar sobre su tema (Rancière, 2011: 15).

La política consiste en la capacidad argumentativa, este hecho es lo que diferencia a los sujetos, desde la antigüedad, dotándolos de las habilidades para poner en tela de juicio lo "justo y lo injusto", como lo menciona el autor, la cuestión es:

[...] saber quién es apto para juzgar lo que es palabra deliberativa y lo que es expresión de desagrado. En cierto sentido, toda la actividad política es un conflicto para decidir qué es palabra o grito, para volver a trazar las fronteras sensibles con las que se certifica la capacidad política (Rancière, 2011: 16).

En la capacidad de decidir los matices de lo debido y lo indebido, lo pertinente y lo impertinente, en eso es donde se reconoce la política, y es en ese espacio donde se articula con la cultura, pues las prácticas sociales son las que caracterizan e identifican a los grupos humanos y permiten su reconocimiento basado en las distinciones que se realizan con la política.

Esa distribución y esa redistribución de los espacios y los tiempos, de los lugares y las identidades, de la palabra y el ruido, de lo visible y lo invisible, conforman lo que llamo el reparto de lo sensible. La actividad política, reconfigura el reparto de lo sensible. Pone en escena lo común de los objetos y de los sujetos nuevos. Hace visible lo que era invisible, hace audibles cual seres parlantes a aquellos que no eran oídos sino como animales ruidosos (Rancière, 2011: 16).

La decisión sobre esa distribución y redistribución es la que está en pugna cuando se generan conflictos políticos y es la que queda de manifiesto con la cultura. 
En el Espectador emancipado, Rancière pone de manifiesto la manera en la que opera el discurso al exponer la contradicción en las prácticas culturales de las protestas de Mayo del 68:

La bestia, dicen, ejerce su influencia sobre los deseos y las capacidades de sus enemigos potenciales ofreciéndoles al mejor precio la más preciada de las mercancías: la capacidad de experimentar la propia vida como un espacio de infinitas posibilidades. Así le ofrece a cada uno lo que él puede anhelar, reality shows para los cretinos y posibilidades acrecentadas de autovalorización para los pícaros. Ésa, nos dice el discurso melancólico es la trampa en la que han caído aquellos que creían derribar el poder capitalista y le han dado al contrario los medios para rejuvenecerse nutriéndose de las energías contestatarias (Rancière, 2010: 38 ).

Es en estas formas de reconocimiento de la realidad en las que las experiencias del observador de segundo orden entran en el juego de la paradoja del valor de los objetos simbólicos, en los que se hacen evidentes aspectos como la condición de clase, el status o la superioridad económica, que observamos de manera lamentablemente cotidiana en las tácticas de los grupos de dominación, y que se aprovechan de su capacidad de manejo de los discursos para darle un determinado sentido a la realidad, convirtiendo todo acto emancipatorio en un espectáculo que actúa a su favor.

Por tal razón, es necesario evaluar las prácticas culturales que se dan en la ciudad desde las experiencias estéticas, considerando para ello el funcionamiento del discurso en el que el desacuerdo es un punto fundamental del campo de operación de la política.

Por desacuerdo se entenderá un tipo determinado de situación de habla: aquella en la que uno de los interlocutores entiende y a la vez no entiende lo que dice el otro. El desacuerdo no es el conflicto entre quien dice blanco y quien dice negro. Es el existente entre quien dice blanco y quien dice blanco pero no entiende lo mismo o no entiende que el otro dice lo mismo con el nombre de la blancura (Rancière, 1996: 8).

Siguiendo a Foucault y a Rancière consideramos a la resistencia como un tipo de desacuerdo. Por lo tanto, en dicho desacuerdo uno de los interlocutores, el que no tiene la palabra, el que no es dueño del discurso es quien se resiste a entender lo mismo que su interlocutor le pretende imponer en una práctica cultural estética. Rancière especifica que este desacuerdo, al que nosotros le denominamos tolerante, combativo, en oposición a la acción o violencia del otro, superviviente, durable, que se opone con fuerza, que rechaza y que no comprende pues opone dificultades para comprender la realidad de la manera en la que se le impone y no por desconocimiento sino con la plena conciencia de causa de lo realizado.

El desacuerdo no es el desconocimiento. El concepto de desconocimiento supone que uno $\mathrm{u}$ otro de los interlocutores, ambos por el efecto de una simple ignorancia, de un disimulo concertado o de una ilusión constitutiva no saben lo que dicen o lo que dice el otro (Rancière, 1996: 8).

La experiencia por parte del interlocutor puede ser fácilmente tachada de haber sido malinterpretada, y de haberse suscitado un malentendido. En el desacuerdo, nos menciona el autor, no cabe el malentendido, al contrario, este mecanismo puede ser utilizado para confundir a quien se resiste y boicotear su posición de resistencia.

[El desacuerdo] Tampoco es el malentendido que descansa en la imprecisión de las palabras. Una antigua cordura hoy particularmente estimada deplora que se entienda mal porque las palabras 
intercambiadas son equívocas. Y reclama que, al menos allí donde estén en juego lo verdadero, el bien y lo justo, se trate de atribuir a cada palabra un sentido bien definido que la separe de las demás, abandonando las que no designan ninguna propiedad definida o las que no pueden escapar a la confusión homonímica (Rancière, 1996: 8).

Como hemos podido constatar el campo de la política implica una serie de elementos y definiciones que nos permitan ir articulando el fenómeno de las prácticas culturales en el contexto de la experiencia estética de la ciudad; sin embargo, es necesario explicar la manera como operan el método con el que se podrá comprender el funcionamiento de la cultura que, además del conocimiento, está en la base de la experiencia estética.

\section{Prácticas culturales, táctica y estrategia. De Certeau y el con- cepto de cultura}

La cultura es una creación, la creación se hace en lo cotidiano. Siguiendo a Maturana no podríamos pensar en lo cotidiano como una prescripción ya dada, previamente elaborada, si lo hiciéramos de esta manera estaríamos hablando de otra cosa, no de cultura. Pero, ¿qué es lo cotidiano?, y sobre todo, ¿cómo definimos la prácticas culturales en relación a este concepto?

De Certeau, desde su perspectiva de estudioso de la cultura, transdisciplinario, complejo y muy peculiar en su abordaje teórico que lo sitúa lejos del campo de la historia, de la antropología y de la filosofía, pero muy cercano a todas ellas por el tipo de problemas que estudia, en su texto La invención de lo cotidiano (2006) nos define qué son las prácticas culturales, en el contexto de lo cotidiano, y lo hace a través de dos conceptos que para explicar la experiencia estética en la ciudad son relevantes: la táctica y la estrategia.
En este documento el autor se concentra en explicar la forma en la que la cultura se configura a través de la performatividad de los acontecimientos dados en la realidad. Esa praxis de la que Maturana hablaba, que permite considerar las características de un sujeto biológico, que tiene clara la contradicción en la paradoja de la existencia, y que se sabe y se reconoce como perteneciente a un espacio y a un tiempo histórico. Habitualmente, dice el autor que la teoría se concentra en la descripción de elementos que son requeridos por las "cuadrículas institucionales" información protocolaria en este tipo de trabajos, los cuales que se hacen en la normatividad de los modelos académicos e institucionales: "[...] cuenta lo que es utilizado, no las maneras de utilizarlo. Paradójicamente, éstas se vuelven invisibles en el universo de la codificación y de la transparencia generalizadas" (De Certeau, 2006: 41).

Lo que interesa en las prácticas culturales es la explicación, para su comprensión, de las operaciones y los funcionamientos del discurso y del lenguaje de la estética, que permite el conocimiento de la ciudad. Para lograr esta finalidad es necesario indagar la manera en la que operan las instituciones y los grupos sociales en relación con este fenómeno.

Llamo estrategia al cálculo [o a la manipulación] de las relaciones de fuerzas que se hace posible desde que un sujeto de voluntad y de poder [una empresa, un ejército, una ciudad, una institución científica] resulta aislable. La estrategia postula un lugar susceptible de ser circunscrito como algo propio y de ser la base donde administrar las relaciones con una exterioridad de metas o de amenazas [los clientes o los competidores, los enemigos, el campo alrededor de la ciudad, los objetivos y los objetos de la investigación, etcétera]. Como en la administración gerencial, toda racionalización "estratégica" se ocupa primero de distinguir en un "medio ambiente" lo que es "propio", es decir, el lugar del poder y de la voluntad propios. Acción cartesiana, si se quiere: 
circunscribir lo propio en un mundo hechizado por los poderes invisibles del Otro. Acción de la modernidad científica, política o militar (De Certeau, 2006: 42).

En las prácticas culturales la estrategia es la manipulación de las relaciones de fuerzas para que desde el sujeto de voluntad y de poder se postulen discursos, y que las experiencias de la ciudad queden como algo propio. Habría que identificar cuáles son las estrategias, y de acuerdo con lo formulado cuál es la resistencia que se presenta ante los mecanismos discursivos estratégicos formulados por los sujetos de poder. También habría que identificar los desacuerdos y malentendidos que se establecen como parte del funcionamiento de las prácticas culturales.

Para hablar de resistencia De Certeau utiliza la metáfora de la táctica, "es un arte del débil", menciona el autor; por su carácter furtivo y supeditado a la línea propuesta por la estrategia, pero finalmente es un acto de desacuerdo con el otro, a sabiendas de que si se realiza un enfrentamiento abierto existe la posibilidad de sucumbir ante la potencia del poderoso, por lo que más vale utilizar los recursos disponibles para lograr los objetivos planteados.

En relación con las estrategias [cuyas figuras sucesivas desplazan este esquema demasiado formal y del cual el vínculo con una configuración histórica particular de la racionalidad estaría por precisarse], llamo táctica a la acción calculada que determina la ausencia de un lugar propio. Por tanto ninguna delimitación de la exterioridad le proporciona una condición de autonomía. La táctica no tiene más lugar que el del otro [...] No cuenta pues con la posibilidad de darse un proyecto global ni de totalizar al adversario en un espacio distinto, visible y capaz de hacerse objetivo. Obra poco a poco. Aprovecha las "ocasiones" y depende de ellas, sin base donde acumular los beneficios, aumentar lo propio y prever las salidas. No guarda lo que gana. Este no lugar le permite, sin duda, la movilidad, pero con una docilidad respecto a los azares del tiempo, para tomar al vuelo las posibilidades que ofrece el instante. Necesita utilizar, vigilante, las fallas que las coyunturas particulares abren en la vigilancia del poder propietario. Caza furtivamente. Crea sorpresas. Le resulta posible estar allí donde no se le espera. Es astuta (De Certeau, 2006: 43).

La táctica, entonces, es una operación dentro de la misma estrategia para subvertir el orden imperante sin ser expulsado del régimen. Esta manera de actuar por mínima acción, posibilita darle un espacio a la resistencia en las prácticas culturales frente al poder omnipotente de las estrategias.

\section{A manera de conclusión}

La tarea a la que nos enfrentamos al hablar de las prácticas culturales en la ciudad no es el reconocimiento de las tácticas, sino su listado estadístico y sistemático, pues eso es otro tipo de información que no proporciona el reconocimiento de las tácticas, por lo menos identificar la forma en la que operan y tratar de señalar algunos de los rastros que van dejando en el tiempo y el espacio.

Pensamos en la idea del lenguaje de una manera distinta a la visión estructuralista, que da cuenta de las estructuras subyacentes en el lenguaje y de las reglas que permiten la comprensión del funcionamiento del mismo. De Certeau sigue los postulados de Wittgenstein para centrar su propuesta en la idea del lenguaje y del discurso dentro del lenguaje ordinario, como un conjunto de prácticas en las que el actor y el acontecimiento encuentran involucrados y a través de las cuales se entiende el mundo.

Las prácticas culturales en las que la experiencia estética y política de la ciudad se realizan son, entonces, un orden del discurso, pero lejos de lo que enuncia Foucault, no las reconocemos como institucionalizadas por los mecanismos de poder 
ni por las estrategias de resistencia, sino la operación del discurso se da a nivel de lo cotidiano en donde podemos observar la forma en la que la táctica opera. Como lo mencionamos anteriormente, desde la perspectiva de la complejidad de Morin, es un problema complejo, recursivo, en múltiples niveles de realidad, en fuga e inasible, por lo que será imposible la enumeración de un listado de prácticas. Nuestra tarea será dar los elementos que sirvan para comprender el fenómeno y dotar al interlocutor de la posibilidad de reconocerlo cuando realice un recorrido por la ciudad.

\section{REFERENCIAS}

Bejarano, A. (2010). "Estética y política en Jacques Rancière. Genealogías de una obra en curso". En Revista de Estudios Sociales, núm. 35. Bogotá, Colombia: Universidad de los Andes. De Certeau, M. (2006). La invención de lo cotidiano 1. Artes de hacer. México: Universidad Iberoamericana.

Foucault, M. (1972). La arqueología del saber. México: Siglo XXI. Giraldo, R. (2006). "Poder y resistencia en Michel Foucault". En Tabula Rasa. Disponible en: https://www.redalyc.org/articulo. oa?id=396/39600406 [Consultado el 24 de octubre de 2019].

Haidar, J. (2006). Debate CEU-Rectoría. Torbellino pasional de los argumentos. México: UNAM.

Homobono, J. I. (2003). "La ciudad y su cultura, en la obra de Lewis Mumford". En Zainak. Cuadernos de Antropología-Etnografía, núm. 23.

Maturana, H. (2002). La objetividad. Un argumento para obligar. Madrid, España: Océano-Dolmen.

Morin, E. (1994). El método 1. La naturaleza de la naturaleza. España: Cátedra. Morin, E. (2004). "La epistemología de la complejidad". En Gazeta de Antropología. Disponible en: https://www.ugr. es/ pwlac/G2O_o2Edgar_Morin.pdf [Consultado el 28 de noviembre de 2018].

Morin, E. (2009). Introducción al pensamiento complejo. España: Gedisa.

Nicolescu, B. (s.a.). La transdisciplina. Manifiesto. Mónaco: Du Rocher.

RAE (2018). Diccionario de la Real Academia Española. Disponible en: http://lema.rae.es/drae2oo1/srv/ search?id=ACXWYAGNYDXX2V40PcNh [Consultado el o8 de diciembre de 2018].

Rancière, J.(1996). El desacuerdo. Buenos Aires, Argentina. Nueva Visión. 
ARTÍ́CULO • La resistencia desde el arte como práctica cultural en la ciudad • OLIVIA F RAGoso susUNAGA

Rancière, J. (2010). El espectador emancipado. Buenos Aires,

Argentina: Manantial.

Rancière, J. (2011). Política de la literatura. Buenos Aires, Argentina:

Libros del Zorzal. 